\title{
Reactivity of hydroxy-containing aromatic compounds towards electrogenerated hydroxyl radicals
}

Raquel Oliveira, Nelson Pereira, Dulce Geraldo, Fátima Bento*

Centro de Química, Universidade do Minho, Campus de Gualtar 4710-057 Braga, Portugal

* Corresponding author Tel.: +351 253604399; fax: +351 253604382; e-mail: fbento@quimica.uminho.pt

\begin{abstract}
A kinetic study on the oxidation of hydroxy-containing aromatic compounds by electrogenerated $\mathrm{HO}$ radical and simultaneous by direct electron transfer is presented. First order kinetics are used to describe consumption rates of hydroquinone, benzoic acid and of hydroxybenzoic acid derivatives by galvanostatic electrolysis with simultaneous oxygen evolution at a Pt electrode. Linear correlations were established from the effect of electrolyses current density on $k_{a p p}$. The meaning of the intercept and of the slope is analysed. A good agreement is found between intercept values and the apparent rate constants from potentiostatic electrolysis without $\mathrm{O}_{2}$ evolution. Simultaneously, the slopes magnitude corroborate the relative reactivity order of species that was established considering the occurrence of positive charge densities on carbon atoms of the aromatic ring. Therefore, the present analysis provides kinetic information concerning both, the direct electron-transfer and the reaction with HO radical.
\end{abstract}




\section{Keywords:}

Electrochemical oxidation; Hydroxyl radical; Hydroxybenzoic acid derivatives; Hydroquinone; Platinum

\section{Introduction}

Electrochemical methods have proved to be adequate for the destruction of organics in aqueous media. A significant number of researchers has sought new electrode materials and improved reactors design to optimize mineralization indexes. Boron-doped diamond (BDD) was the most used anode material for this purpose [1-7], allowing to an efficient decrease of the TOC (total organic content) of aqueous solutions containing test compounds, such as phenol [8,9], benzoic acid [10,11], bisphenol A [12] or gallic acid [13]. The efficiency of organics destruction, by means of their electrooxidation with simultaneous oxygen evolution, was attributed to the formation of HO radicals as intermediaries of water electrooxidation [14-16]. The formation of this radical was detected in assays using anodes of $\mathrm{Ti} / \mathrm{IrO}_{2}, \mathrm{Ti} / \mathrm{SnO}_{2}$ and $\mathrm{Pt}$ using a radical trap [15]. The following mechanism was proposed for the oxidation of organics mediated by $\mathrm{HO}$ radicals [17].

$$
\begin{aligned}
& \mathrm{H}_{2} \mathrm{O} \stackrel{k_{\mathrm{HO}}}{\longrightarrow} \mathrm{HO}^{\bullet}+\mathrm{H}^{+}+\mathrm{e}^{-} \\
& 2 \mathrm{HO} \stackrel{k_{O_{2}}}{\longrightarrow} \mathrm{O}_{2}+2 \mathrm{H}^{+}+2 \mathrm{e}^{-} \\
& \mathrm{R}+\mathrm{n} \mathrm{HO}^{\cdot} \stackrel{k_{R, H O}}{\longrightarrow} \text { Products }
\end{aligned}
$$

The adsorption of $\mathrm{HO}$ radicals at the anode surface has a significant effect on their reactivity. When they are strongly adsorbed they display lower reactivity, as it happens 
for $\mathrm{Pt}$ or $\mathrm{IrO}_{2}$, whereas when the adsorption strength is weak, as it happens for BDD, the reactivity of electrogenerated radicals is important [7].

Although most of the available studies deal with high oxidation power anodes, the use of anodes with low oxidation power can have important applications particularly when a certain degree of selectivity is required for oxidation.

In a previous work [18] we have reported a kinetic study on the oxidation of two compounds (BA and 4-HBA) using BDD and Pt, where it was shown that consumption of species occurred mainly by reaction with electrogenerated HO radicals. Based on these results, a kinetic treatment was presented, considering the relative magnitude of the organics concentration towards $k_{R, H O} / k_{O_{2}}$, that allows the interpretation of concentrations decay during galvanostatic electrolysis.

In this work, a kinetic study on aromatic compounds oxidation by electrogenerated HO radical with simultaneous direct electron transfer is presented. The possibility of extending our previous analysis to electroactive compounds is quite relevant as a great number of organic compounds is electroactive, particularly the model compounds used in mineralization studies. Compounds used in this work were selected regarding the direct electron transfer reaction features namely, the number of electrons involved (one or two) and the stability of the formed products (semiquinone radical or quinones). Kinetic data is analysed considering the presumed reactivity of these species suggested from charge density values on carbon atoms of the aromatic ring. 


\section{Experimental}

\subsection{Chemicals}

All reagents employed were of analytical grade: benzoic acid (BA; Prolabo), 4hydroxybenzoic acid (4-HBA; BDH Chemicals), 2,3-dihydroxybenzoic acid (2,3-HBA; ACROS Organics), 2,4-dihydroxybenzoic acid (2,4-HBA; ACROS Organics), 2,5dihydroxybenzoic acid (2,5-HBA; ACROS Organics), 3,4,5-trihydroxybenzoic acid (3,4,5-HBA; Sigma), hydroquinone (HQ; May \& Baker, Ltd), potassium chloride (Fluka), potassium ferrocyanide and potassium ferricyanide (José Gomes Santos), potassium dihydrogen phosphate and phosphoric acid (ACROS Organics). Methanol was of HPLC grade from Fisher Scientific.

\subsection{HPLC}

Oxidation reactions were monitored following the concentration decrease along galvanostatic electrolyses by HPLC. HPLC experiments were performed using a Jasco, PU-2080 Plus system equipped with a RP 18 column from Grace Smart $(250 \mathrm{~mm} \times 4.6$ mm, $5 \mu \mathrm{m}$ particle size) and using Clarity HPLC software from Jasco (Jasco 870 / UV

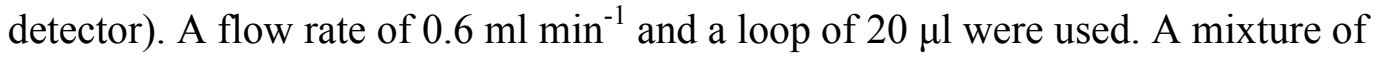
methanol, water and phosphoric acid (60:39:1) (v/v) was used as mobile phase. The detection wavelength was selected according to species: $210 \mathrm{~nm}$ for 2,3-HBA and 2,4HBA; $230 \mathrm{~nm}$ for BA, 4-HBA and 2,5-HBA; and $280 \mathrm{~nm}$ for 3,4,5-HBA and HQ. The quantification was performed using calibration curves. 


\subsection{Electrochemical measurements}

Voltammetric measurements and galvanostatic / potentiostatic electrolyses were performed using a potentiostat (Autolab type PGSTAT30, Ecochemie) controlled by GPES 4.9 software provided by Ecochemie.

\subsubsection{Cyclic voltammetry}

Cyclic voltammetry experiments were carried out from -0.25 to $1.4 \mathrm{~V}$ using an undivided three-electrode cell. The working electrodes were of glassy carbon (GC; 3 mm diameter disk electrode, $\mathrm{CHI} 104, \mathrm{CH}$ Instruments, Inc.) and of Pt (EM-EDI, Radiometer Analytical). An Ag/AgCl, 3.0 M (CHI111, CH Instruments, Inc.) was used as reference electrode and a Pt wire as counter electrode. The surface of the GC electrode was cleaned between scans by polishing with polycrystalline diamond suspension ( $3 \mathrm{~F} \mu \mathrm{m}$; Buehler) for $\approx 1 \mathrm{~min}$. The Pt electrode was electrochemically cleaned in $0.10 \mathrm{M}$ phosphate buffer $\mathrm{pH} 3.5$ at the oxygen evolution region $(0.02 \mathrm{~A})$ during $600 \mathrm{~s}$.

\subsubsection{Electrolysis}

Galvanostatic electrolyses were carried out using current densities from 50 to $1250 \mathrm{~A} \mathrm{~m}^{-}$

${ }^{2}$ in a two compartments cell separated by a glass frit membrane. The volume of the anodic compartment was $9.0 \mathrm{ml}$ and the solution was mechanically stirred with a magnetic stir bar $(300 \mathrm{rpm})$. The anode is made of a piece $(20 \mathrm{~mm} \times 10 \mathrm{~mm}) \mathrm{of} \mathrm{Pt}$ gauze (52 mesh woven from $0.1 \mathrm{~mm}$ diameter wire, 99.9\%, from Alfa Aesar). Before each experiment the anode was cleaned electrochemically in $0.1 \mathrm{M}$ phosphate buffer $\mathrm{pH}$ 3.5 during $600 \mathrm{~s}$ at a constant current of $0.02 \mathrm{~A}$. The area of the Pt working electrode $\left(5.6 \mathrm{~cm}^{2}\right)$ was determined in a chronoamperometry experiment using $1.00 \mathrm{mM}$ of $\mathrm{K}_{3}\left[\mathrm{Fe}(\mathrm{CN})_{6}\right]$ in $0.1 \mathrm{M} \mathrm{KCl}[19]$. 
Reported apparent rate constants from oxidation of hydroxybenzoic acid derivatives and of HQ were determined using data of at least two electrolyses and displayed uncertainties correspond to standard deviations.

\subsection{Diffusion coefficients}

Diffusion coefficients $(D)$ were estimated from the slope of $I_{p}$ vs. $v^{1 / 2}$ (regarding voltammetric data from 20 to $100 \mathrm{mV} \mathrm{s}^{-1}$ ) for hydroxybenzoic acids derivatives whose first oxidation peaks involve a single electron. $\alpha$ values were estimated considering $\left(E_{p^{-}}\right.$ $\left.E_{p / 2}\right)=48 /(\alpha n)$ (Table 1). The number of electrons of the first oxidation peak in Table 1 were obtained from literature [20-23]. As the homogeneous rate constants were not known, the selection of the scan rates was based on the fit to a linear dependence of $I_{p}$ with $v^{1 / 2}$. In this way, linearity between $I_{p}$ and $v^{1 / 2}$ at the higher scan rates confirms that the contribution of double layer charging current is negligible vis a vis the faradaic current as double layer charging current varies linearly with $v$ and not with $v^{1 / 2}$. On the other hand, pure kinetic behaviour (lower scan rates) was discarded as in this region $I_{p}$ of EC processes changes only slightly with $v^{1 / 2}$ [24]. Despite a pure diffusion behaviour is not assured the introduced uncertainty is known to be low for EC processes [24]. Validation of determined D values cannot be performed as there are not available $D$ values for most of the compounds analysed. For 3,4,5-HBA the calculated value of $D$ is in agreement with that reported elsewhere based on simulation results [22] with a deviation of 5\%. Reported uncertainties were calculated using the standard deviation of the slope of $I_{p}$ vs. $v^{1 / 2}$. 
Table 1: Voltammetric data (regarding the first oxidation peak) of the different hydroxybenzoic acid derivatives, hydroquinone and of potassium hexacyanoferrate estimated from results in Figure 1. Diffusion coefficients of species whose first oxidation peak involves a single electron were determined by from the slope of $I_{p}$ vs. $v^{1 / 2}$.

\begin{tabular}{|c|c|c|c|c|c|c|c|}
\hline & $E_{p}(\mathrm{~V})$ & $I_{p}\left(10^{-6} \mathrm{~A}\right)$ & $E_{p}-E_{p / 2}(\mathrm{mV})$ & $E_{p}^{a}-E_{p}^{c}(\mathrm{mV})$ & $n$ & $\alpha$ & $D\left(10^{-5} \mathrm{~cm}^{2} \mathrm{~s}^{-1}\right)$ \\
\hline 4-HBA & $1.002 \pm 0.004$ & $7.36 \pm 0.02$ & 78 & - & $1[20]$ & 0.62 & $3.5 \pm 0.1$ \\
\hline 2,3-HBA & $0.496 \pm 0.007$ & $10.54 \pm 0.07$ & 69 & - & $1[21]$ & 0.70 & $3.6 \pm 0.6$ \\
\hline 2,4-HBA & $0.996 \pm 0.006$ & $9.56 \pm 0.04$ & 74 & - & $1[20]$ & 0.65 & $3.8 \pm 0.3$ \\
\hline 2,5-HBA & $0.430 \pm 0.004$ & $8.75 \pm 0.04$ & 51 & 135 & $2[27]$ & a) & - \\
\hline 3,4,5-HBA & $0.527 \pm 0.001$ & $7.72 \pm 0.03$ & 73 & - & $1[22]$ & 0.66 & $3.7 \pm 0.2$ \\
\hline HQ & $0.477 \pm 0.004$ & $5.91 \pm 0.03$ & 71 & 288 & $2[23]$ & a) & - \\
\hline$\left[\mathrm{Fe}(\mathrm{CN})_{6}\right]^{4-}$ & $0.281 \pm 0.001$ & $3.07 \pm 0.02$ & 60 & 65 & $1[24]$ & b) & $0.77 \pm 0.03$ \\
\hline
\end{tabular}

a) ECEC mechanism

b) reversible electron transfer

2.5. Hydrodynamics characterization of the electrolysis cell

The mass transport efficiency of the electrochemical cell was characterized by analysis of $j$ - $t$ curves from electrolyses $(1.2 \mathrm{~V})$ of $0.50 \mathrm{mM} \mathrm{K} 4\left[\mathrm{Fe}(\mathrm{CN})_{6}\right]$ in $0.15 \mathrm{M}$ phosphate buffer pH 3.5 (Eq. (4)) [24] .

$\frac{j}{j_{0}}=\exp \left(-\frac{k_{a p p} A}{V} t\right)$

where, $A$ is the anode surface area, $V$ is the volume of the solution in the anodic compartment, $k_{\text {app }}$ is the apparent rate constant that characterizes the consumption of the substrate and $t$ is time. As oxidation of $\left[\mathrm{Fe}(\mathrm{CN})_{6}\right]^{4-}$ is a very fast one-electron transfer, the process is mass transport controlled and therefore $k_{\text {app }}=k_{m}$ :

$k_{m}=\frac{D}{\delta}$

where, $k_{m}$ is the mass transport coefficient and $\delta$ is the diffusion-layer thickness. 
From Eq. (5) $\delta=2.53 \times 10^{-3} \mathrm{~cm}$ was determined using $k_{m}=3.04 \times 10^{-3} \mathrm{~cm} \mathrm{~s}^{-1}$

(evaluated from $j-t$ curve of potentiostatic electrolysis) and $D=7.7 \times 10^{-6} \mathrm{~cm}^{2} \mathrm{~s}^{-1}$ (from voltammograms recorded in $0.15 \mathrm{M}$ phosphate buffer $\mathrm{pH} 3.5$ and using Cottrell equation). Calculated values of $D$ for $\left[\mathrm{Fe}(\mathrm{CN})_{6}\right]^{4-}$ is in agreement with that reported $[25]$.

2.6. Charge density calculations

Charge density values were calculated using MarvinSketch, a Java based chemical editor, provided by platform ChemAxon. Representation of molecules was drawn also using MarvinSketch.

\section{Results and discussion}

Electrogeneration of $\mathrm{HO}$ radicals from water occurs at potentials higher than those required for oxidation of most hydroxybenzoic acid derivatives, therefore it is expected that their direct oxidation occurs simultaneously with the oxidation via HO radicals. Characterization of voltammetric response of these compounds is consequently relevant for interpretation of their oxidation kinetics.

\subsection{Cyclic voltammetry and potentiostatic electrolysis}

Cyclic voltammetry of 4-HBA, 2,3-HBA, 2,4-HBA, 2,5-HBA, 3,4,5-HBA and HQ in phosphate buffer $\mathrm{pH} 3.5$ was carried out at Pt and GC electrodes. Voltammograms of BA (both at Pt and at GC) and of 4-HBA (at Pt) are not significantly different from those of blank solution. For the other compounds, voltammograms recorded at $\mathrm{Pt}$ electrode are not well defined due to Pt oxide formation current (not shown). Fig. 1 reports voltammetric responses of 4-HBA, 2,3-HBA, 2,4-HBA, 2,5-HBA and 3,4,5- 
HBA at a GC electrode in phosphate buffer $\mathrm{pH}$ 3.5. Voltammogram of HQ is also reported in Fig. 1 as a reference compound. Table 1 contains voltammetric data from the first oxidation process $\left(I_{p}, E_{p}, E_{p}-E_{p / 2}\right.$ and $\left.E_{p}{ }^{a}-E_{p}{ }^{c}\right)$ as well as experimental values of $D$ (section 2.4).Oxidation of the monohydroxybenzoic acid, 4-HBA, corresponds to a single electron transfer [20]. The peak potential, $E_{p}$, is very positive as compared to HQ peak potential and no significant reverse peak is noticeable. The oxidation process is assigned to the formation of a semiquinone and is accomplished by the abstraction of a proton. The semiquinone radical is very unstable and therefore its formation is followed by other reactions, including dimerization and polymerization $[20,26]$.

Polyhydroxybenzoic acids with two or more HO groups can be oxidized by one or more electrons. This is clearly observed in the voltamograms of 2,3-HBA and of 3,4,5-HBA where the $\mathrm{HO}$ groups are in ortho position in respect to each other. In this configuration the semiquinone radical formed by the first electron-transfer reaction is further oxidized to quinone in a second process $[21,22]$. In this case the first oxidation is rather facilitated and occurs at a low potential, comparable to that of HQ. Nevertheless, no reverse peak is observed. Voltammogram of 2,4-HBA also displays two peaks (partially overlapped) that can correspond to two successive electron transfer processes. The first oxidation peak occurs at a potential comparable to that of 4-HBA and superior to that of 2,3-HBA and to that of 3,4,5-HBA. The HO groups in 2,5-HBA are located in para position in respect to each other, like in HQ and both compounds are oxidized in a single step involving the transfer of 2 electrons accomplished by the abstraction of 2 protons $[23,27]$. Although the processes are irreversible by an electrochemical perspective the formed quinone can be reduced back in the reverse scan. As global trend, it can be remarked that voltammograms of species that enable the formation of a para-quinone (as for 2,5-HBA and HQ) display a single oxidation 
process with a reverse peak, while when a ortho-quinone can be formed oxidation occurs by two one-electron processes and without reverse peak (as for 2,3-HBA and 3,4,5-HBA). In both cases (formation of ortho or para-quinone) the first peak potential is rather low compared to that of species that do not afford the formation of quinones (as for 4-HBA and 2,4-HBA).

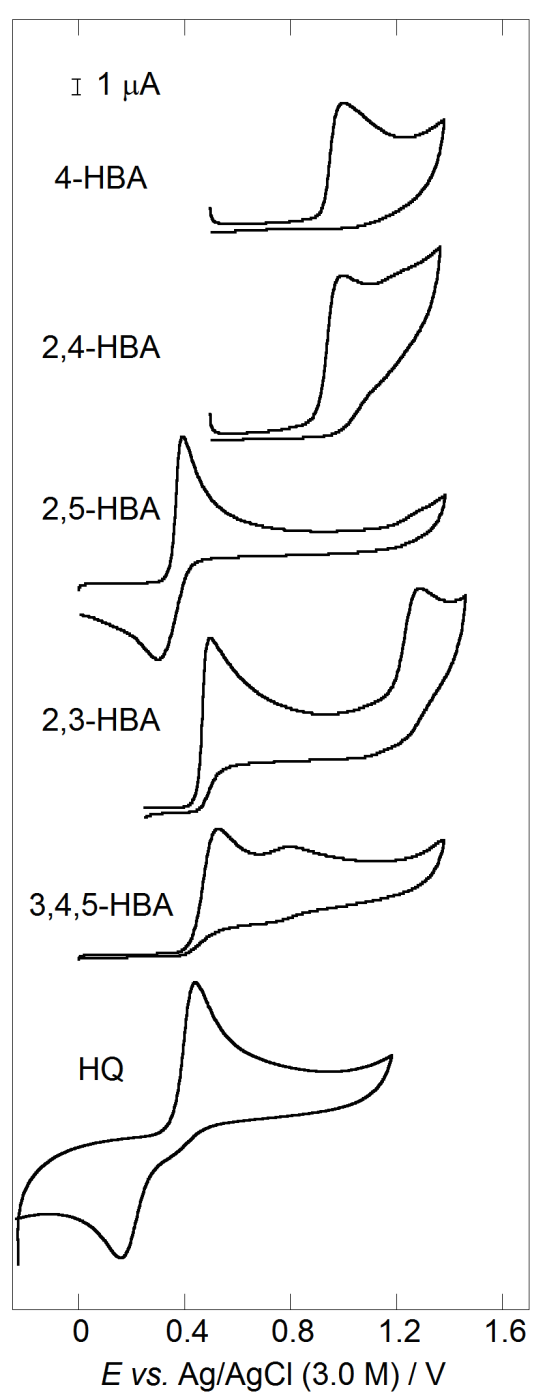

Fig. 1: Cyclic voltammograms (from top to bottom) of $0.50 \mathrm{mM}$ 4-HBA, 2,4-HBA, 2,5-HBA, 2,3-HBA, 3,4,5-HBA and HQ in $0.15 \mathrm{M}$ phosphate buffer solution $\mathrm{pH} 3.5$, recorded at $20 \mathrm{mV} \mathrm{s}^{-1}$ using a GC electrode.

Potentiostatic electrolysis were carried out with BA, 4-HBA, 2,4-HBA, 2,3-HBA, 2,5HBA, 3,4,5-HBA and HQ at $1.2 \mathrm{~V}$. This potential is much higher than the peak 
potential of these species (of voltammograms recorded in carbon electrodes) but is lower than the required for oxygen evolution. Concentration decrease was monitored by HPLC and apparent rate constants were determined according to Eq. (6) that is characteristic of $1^{\text {st }}$ order kinetics:

$$
\frac{C}{C_{0}}=\exp \left(-\frac{k_{a p p} A}{V} t\right)
$$

where, $C$ is the concentration at a given time and $C_{0}$ is the initial concentration. Calculated values for the apparent rate constant for 2,3-HBA, 2,5-HBA, 3,4,5-HBA and HQ are presented in Table 2 as $k_{\text {app }}^{0}$. For BA and for 4-HBA current dropped to zero almost at the start of potentiostatic electrolysis, indicating that electron transfer reaction did not occur at Pt. For 2,4-HBA an abrupt drop of current was observed at the first instants of potentiostatic electrolysis due to anode passivation probably due to formation of polymers at the electrode surface. Values of $k_{m}$ calculated by means of Eq. (5), using $D$ values reported in Table 1, are much higher than experimental $k_{\text {app }}^{0}$ values (Table 2). The difference between these two parameters is quite significant and cannot be assigned to the inaccuracy of $D$ values related to the presence of the coupled chemical reaction as previously discussed. The discrepancy between $k_{m}$ and $k_{\text {app }}^{0}$ provide a strong evidence that the electron transfer rate is low at the present conditions and the electrolysis rate is not limited by mass transport.

\subsection{Galvanostatic electrolysis}

Galvanostatic electrolyses of BA, hydroxybenzoic acid derivatives and of HQ (0.50 $\mathrm{mM}$ ) in $0.15 \mathrm{M}$ phosphate buffer $\mathrm{pH} 3.5$ using Pt anode were conducted at a current density of $1250 \mathrm{~A} \mathrm{~m}^{-2}$ with simultaneous oxygen evolution. Concentration decrease, expressed by means of the concentrations ratio $C / C_{0}$, was quantified by HPLC and is plotted against electrolysis time. Values of concentration decrease of BA and of 3,4,5- 
HBA are presented in Fig. 2. Curves displayed are fitted to experimental data considering Eq. (6).

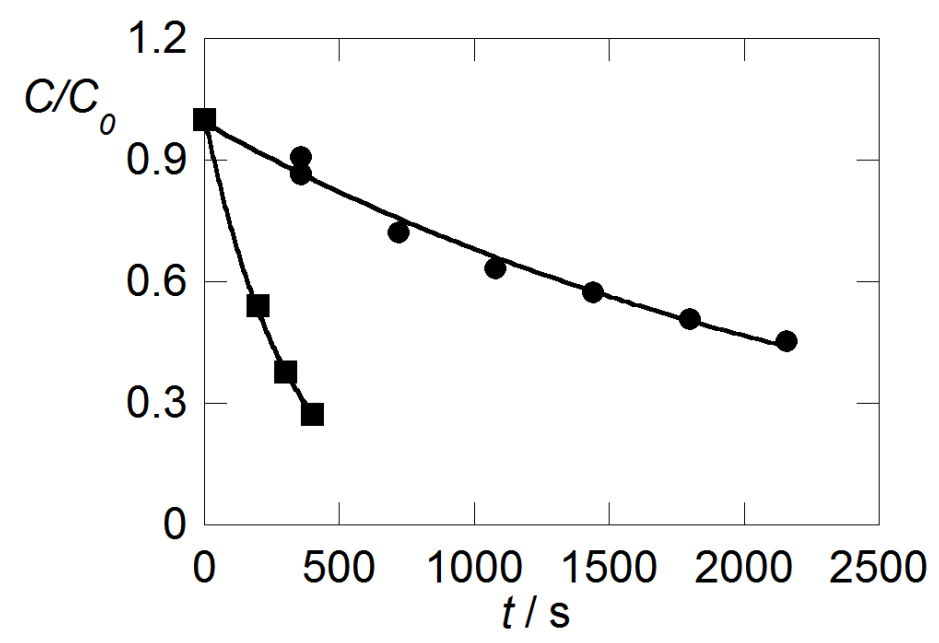

Fig. 2: Concentration decrease during galvanostatic electrolyses (Pt anode at $1250 \mathrm{~A} \mathrm{~m}^{-2}$ ) of $0.50 \mathrm{mM}$ solutions: BA $\left(C / C_{0}=\exp \left((-3.8 \pm 0.1) \times 10^{-4} t\right), \mathrm{r}=0.99\right)$ and $\left.(\boldsymbol{\square}) 3,4,5-\mathrm{HBA}\left(C / C_{0}\right)=\exp \left((-3.6 \pm 0.2) \times 10^{-3} t\right), \mathrm{r}=0.99\right)$. Equations and curves were obtained by regression analysis.

Concentrations decrease of 3,4,5-HBA can be assigned to its oxidation by electrogenerated $\mathrm{HO}$ radicals and also by direct electron-transfer, while for BA is mainly due to oxidation by $\mathrm{HO}$ radicals (as there is no evidence of direct electron transfer by voltammetric studies). Experimental $k_{\text {app }}$ value of $3,4,5-\mathrm{HBA}\left(66 \times 10^{-6} \mathrm{~m} \mathrm{~s}^{-}\right.$ $\left.{ }^{1}\right)$ is quite different from that of BA $\left(6.10 \times 10^{-6} \mathrm{~m} \mathrm{~s}^{-1}\right)$ and is considerable lower than the calculated mass transport coefficient, $k_{m}\left(146 \times 10^{-6} \mathrm{~m} \mathrm{~s}^{-1}\right)$. Values of $k_{m}$ are much higher than experimental $k_{a p p}$ values for all the analyzed hydroxybenzoic acid derivatives (Table 2). If the process was controlled by mass transport $k_{\text {app }}$ values should be higher than the calculated $k_{m}$ values using the $\delta$ obtained from potentiostatic electrolyses without $\mathrm{O}_{2}$ evolution. Due to bubbles formation, convection is increased and thus $\delta$ must be thinner, what would imply higher mass transport efficiency, in opposition to what is observed. These results provide an unequivocal indication that $k_{a p p}$ 
is not limited by mass transport. Instead $k_{\text {app }}$ provides a measure of the rate of whole oxidation reactions associated to the consumption of species, by direct electron transfer and by electrogenerated $\mathrm{HO}$ radicals.

\subsection{Current density effect}

The effect of current density on the consumption of 2,4-HBA is illustrated in Fig. 3, where $C / C_{0}$ values against time are reported for $50,268,625$ and $1250 \mathrm{~A} \mathrm{~m}^{-2}$. All galvanostatic electrolyses were performed with simultaneous $\mathrm{O}_{2}$ evolution.

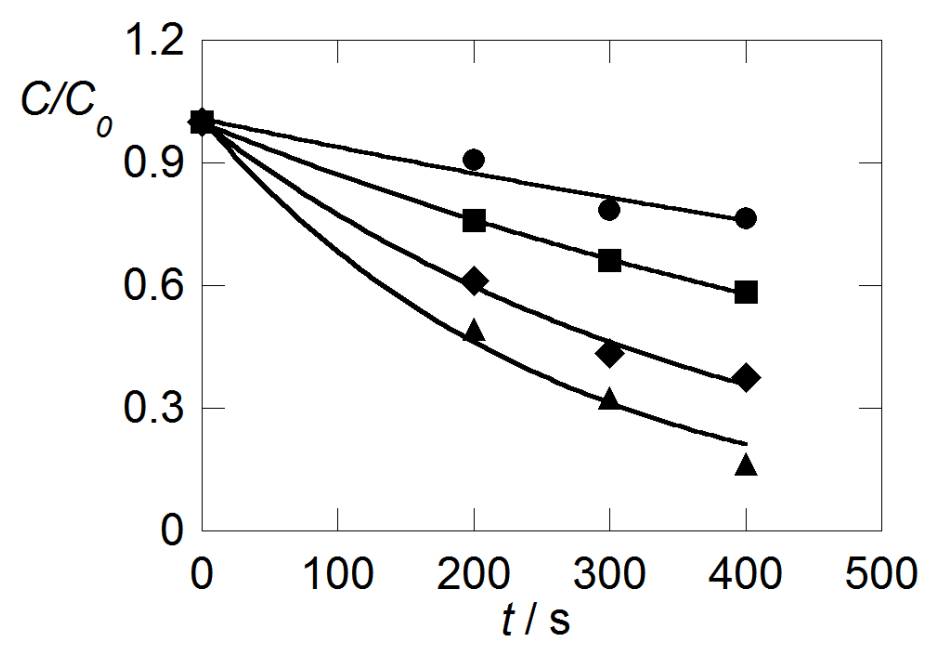

Fig. 3: Concentration decrease during galvanostatic electrolyses of 2,4-HBA $\left(C_{0}=0.50 \mathrm{mM}\right)$ at a Pt anode: $(\bullet) 50 \mathrm{~A}$ $\mathrm{m}^{-2}\left(C / C_{0}=\exp \left((-0.7 \pm 0.1) \times 10^{-3} t\right), \mathrm{r}=0.99\right),(\mathbf{\square}) 268 \mathrm{~A} \mathrm{~m}^{-2},\left(C / C_{0}=\exp \left((-1.36 \pm 0.02) \times 10^{-3} t\right), \mathrm{r}=0.999\right)$, $628 \mathrm{~A} \mathrm{~m}^{-2},\left(C / C_{0}=\exp \left((-2.6 \pm 0.2) \times 10^{-3} t\right), \mathrm{r}=0.99\right)$ and $(\mathbf{\Delta}) 1250 \mathrm{~A} \mathrm{~m}^{-2},\left(C / C_{0}=\exp \left((-3.9 \pm 0.5) \times 10^{-3} t\right), \mathrm{r}=\right.$ 0.99). Equations and curves were obtained by regression analysis.

Experimental $k_{a p p}$ values are reported in Table 2 together with the corresponding values of other hydroxybenzoic acid derivatives and of HQ. An increase of $k_{\text {app }}$ with current density is evident for all the compounds studied. The origin of this variation cannot be attributed to an increase of mass transport rate associated to a raise of oxygen bubbles formation as the processes are not mass transport limited, as explained in the previous 
section. Therefore this variation must be related to the kinetic variables that control the oxidation rates of these compounds.

Table 2: Apparent rate constants from potentiostatic electrolysis $\left(k_{a p p}^{0}\right)$ and from galvanostatic electrolysis $\left(k_{a p p}\right)$ at different current densities. Values of $\left(k_{a p p}\right)_{j=0}$ correspond to the intercept of the straight lines in Fig. 4 . The mass transport coefficient values $\left(k_{m}\right)$ reported were calculated using $\delta=2.53 \times 10^{-5} \mathrm{~m}$ (according to results from $\left[\mathrm{Fe}(\mathrm{CN})_{6}\right]^{4-}$ reported in section 2.5.) and the diffusion coefficients of Table 1.

\begin{tabular}{|c|c|c|c|c|c|c|c|}
\hline & $1.2 \mathrm{~V}$ & & $50\left(\mathrm{~A} \mathrm{~m}^{-2}\right)$ & $268\left(\mathrm{~A} \mathrm{~m}^{-2}\right)$ & $625\left(\mathrm{~A} \mathrm{~m}^{-2}\right)$ & $1250\left(\mathrm{~A} \mathrm{~m}^{-2}\right)$ & \\
\hline & $k_{a p p}^{0}\left(10^{-6} \mathrm{~m} \mathrm{~s}^{-1}\right)$ & $\left(k_{a p p}\right)_{j=0}\left(10^{-6} \mathrm{~m} \mathrm{~s}^{-1}\right)$ & & $k_{a p p}(1$ & $\left.-6 \mathrm{~m} \mathrm{~s}^{-1}\right)$ & & $k_{m}\left(10^{-6} \mathrm{~m} \mathrm{~s}^{-1}\right)$ \\
\hline BA & a) & $-0.3 \pm 0.5$ & $0.38 \pm 0.05$ & $1.21 \pm 0.06$ & $3.60 \pm 0.03$ & $6.10 \pm 0.01$ & c) \\
\hline 4-HBA & a) & $-0.1 \pm 1.2$ & $2.57 \pm 0.06$ & $8 \pm 1$ & $24 \pm 4$ & $45 \pm 4$ & $138 \pm 21$ \\
\hline 2,3-HBA & $18.2 \pm 0.6$ & $21 \pm 1$ & $21.1 \pm 0.5$ & $30 \pm 2$ & $37 \pm 4$ & $52 \pm 3$ & $142 \pm 31$ \\
\hline 2,4-HBA & b) & $9.8 \pm 0.2$ & $11 \pm 2$ & $22 \pm 1$ & $41 \pm 3$ & $67 \pm 3$ & $150 \pm 26$ \\
\hline 2,5-HBA & $19.0 \pm 0.5$ & $21.5 \pm 0.7$ & $22 \pm 2$ & $27 \pm 3$ & $34 \pm 3$ & $46 \pm 2$ & d) \\
\hline $3,4,5-\mathrm{HBA}$ & $30 \pm 8$ & $30 \pm 2$ & $32 \pm 2$ & $39 \pm 3$ & $45 \pm 4$ & $66 \pm 6$ & $146 \pm 23$ \\
\hline HQ & $38.2 \pm 0.4$ & $38 \pm 1$ & $39.6 \pm 0.3$ & $47 \pm 1$ & $53 \pm 2$ & $73 \pm 4$ & d) \\
\hline
\end{tabular}
a) no faradaic current was measured
b) not possible to measure due to the anode passivation during potentiostatic electrolysis.
c) not determined as no voltammetric response was obtained.
d) not calculated as the $1^{\text {st }}$ peak corresponds to a ECEC mechanism.

As consumption of hydroxybenzoic acid derivatives can be due to direct electron transfer as well as to reaction with $\mathrm{HO}$ radicals, Eq. (7) must be considered in addition to Eq. (3):

$$
\mathrm{R}+\mathrm{ne} \mathrm{e}^{-} \stackrel{k_{R, e}}{\longrightarrow} \text { Products }
$$

Therefore the rate of consumption of species results from the contribution of these two processes:

$v_{R}=v_{R, H O}+v_{R, e}$ 
where $v_{R, H O}$ is the rate of the reaction with $\mathrm{HO}$ radicals and $v_{R, e}$ is the rate of the oxidation by electron transfer. Eq. (8) can be rewritten as:

$v_{R}=\left(k_{R, H O} \theta \Gamma_{S}+k_{R, e}\right) C_{R}$

where, $k_{R, H O}$ is the apparent rate constant of the reaction with $\mathrm{HO}$ radicals (Eq. (3)), $\theta \Gamma_{s}$ is the $\mathrm{HO}$ radical surface concentration at the anode, $k_{R, e}$ is the electron transfer rate constant (Eq. (7)) and $C_{R}$ is the concentration of the species. Hence $k_{a p p}$ in Eq. (6) corresponds to:

$k_{\text {app }}=k_{R, H O} \theta \Gamma_{s}+k_{R, e}$

Analysis of $k_{a p p}$ variation with current density must take into account the effect of $j$ on each variable in Eq. (10). Whereas $k_{R, H O}$ and $\Gamma_{s}$ (saturation concentration of $\mathrm{HO}$ radicals) should not be affected by current density, the anode coverage degree, $\theta$, and the heterogeneous rate constant $k_{R, e}$ can depend on it.

The heterogeneous rate constant $k_{R, e}$ may increase with current density if the reaction is not diffusion limited as it is the present case. Although in voltammetric experiments a diffusion control regime was achieved for all species for $E>1.04 \mathrm{~V}$, in electrolysis a diffusion control regime was not attained because the diffusion layer is thinner due to forced convection. Therefore the increase of $k_{R, e}$ may occur if the anode potential increases with $j$. Indeed, when current density is varied from 50 to $268 \mathrm{~A} \mathrm{~m}^{-2}$ the potential increase is not negligible in opposition to what happens for the subsequent variations of current density (from 268 to 628 and from 628 to $1250 \mathrm{~A} \mathrm{~m}^{-2}$ ) as the slope of $E$ - $j$ curves tend to zero for $j \geq 268 \mathrm{~A} \mathrm{~m}^{-2}$ (results not shown). Thus variation of $k_{R, e}$ could only explain an increase of $k_{a p p}$ for the lower concentration densities. However, as the augmentation of $k_{\text {app }}$ is considerable for higher current densities, the variation of $k_{R, e}$ cannot be overall justified by $k_{R, e}$ increase.

On the other hand, the rate of formation of HO radicals (Eq. (1)) is controlled by $j$ [28]: 
$v_{H O} \cdot=\frac{j}{z \mathrm{~F}}$

Furthermore, in conditions of low $C_{R}$ it was demonstrated that [18]:

$\theta \Gamma_{s}=\frac{j}{2 z \mathrm{~F} k_{O_{2}}}$

where, $k_{\mathrm{O}_{2}}$ is the rate constant of $\mathrm{O}_{2}$ formation (Eq. (2)).

From Eq. (12) it is expected that the surface concentration of HO radicals increases steadily with $j$. As a consequence of this concentration increase, and based on Eq. (10) it is foreseen a linear variation of $k_{\text {app }}$ with $j$ :

$$
k_{a p p}=\frac{1}{2 z \mathrm{~F}} \frac{k_{R, H O}}{k_{O_{2}}} j+k_{R, e}
$$

The observation of this linear trend implies that $k_{R, e}$ does not vary significantly with $j$.

\subsection{Correlation between apparent rate constant and current density}

In Fig. 4 the apparent rate constant of BA, hydroxybenzoic acid derivatives and of HQ are plotted against current density. Open symbols $\left(k_{\text {app }}^{0}\right)$ correspond to potentiostatic electrolyses $(E=1.2 \mathrm{~V})$, whereas solid symbols $\left(k_{\text {app }}\right)$ correspond to galvanostatic electrolyses. Straight lines were obtained from regression analysis considering only $k_{a p p}$ values.

Plots in Fig. 4 for all species display linear trends of $k_{a p p}$ vs. $j$ as predicted by Eq. (13). The magnitudes of the intercept differ significantly among them. While a null intercept is found for BA and 4-HBA, noteworthy intercepts are found for all the other species. The origin of the intercept can be explained as follows. As current density approaches zero the amount of $\mathrm{O}_{2}$ produced vanishes and no $\mathrm{HO}$ radicals will be generated; therefore the oxidation reaction will only take place by direct electron transfer. The fact that zero intercepts are found for BA and 4-HBA means that no significant consumption 
of these species is observed in the absence of HO radicals, which is consistent with the absence of a voltammetric response at Pt. Besides, the match between the straight lines intercept and $k_{a p p}^{0}$ values from potentiostatic electrolysis (open symbols) provides a clear evidence that the intercept is a measure of the apparent rate constant of oxidation via direct electron transfer. The similarity between the experimental $\left(k_{a p p}^{0}\right)$ and extrapolated $\left(k_{a p p}\right)_{j=0}$ (Fig. 4(a) and Table 2$)$ is also an evidence that $k_{R, e}$ was not significantly affected by current density increase (see section 3.3 ).

The meaning of the slope of $k_{a p p}$ vs. $j$ was thoroughly discussed by us in a previous work considering different conditions (i.e. different magnitude of $C_{R}$ with regard to $\left.k_{\mathrm{O}_{2}} / k_{R, H O}\right)$ [18]. In brief, the slope is a measure of the degree of susceptibility of $k_{\text {app }}$ to an increase of $\mathrm{HO}$ radicals concentration at the anode, that is related to the relative magnitude of $k_{R, H O}$ towards $k_{O_{2}}$.
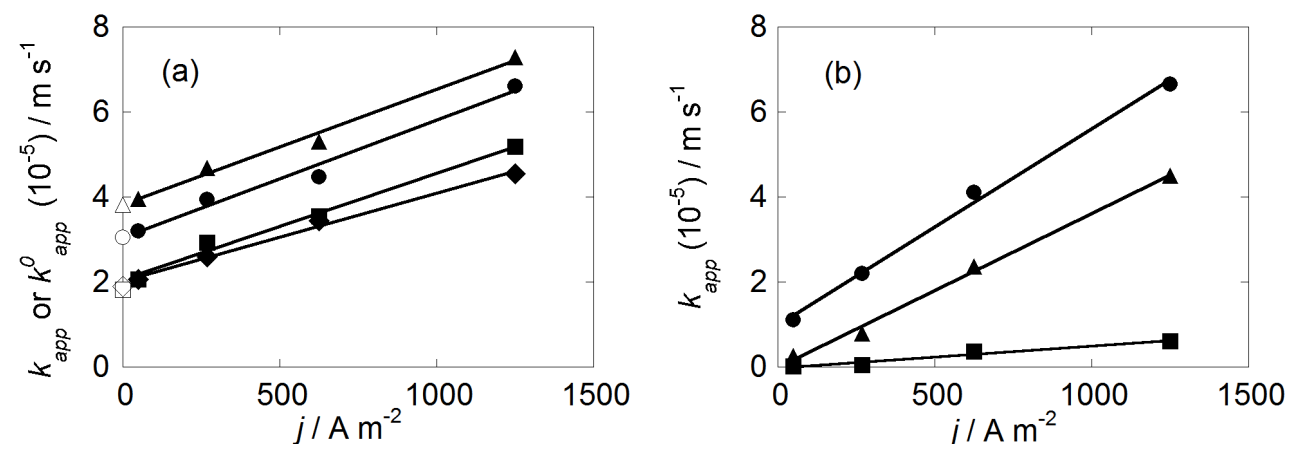

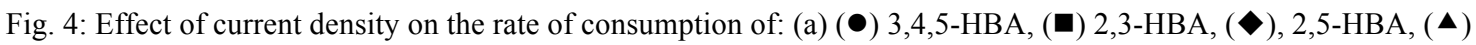

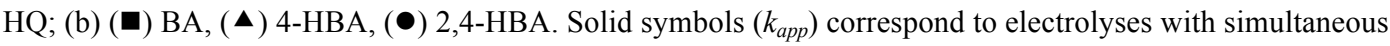
oxygen evolution whereas open symbols $\left(k^{0}{ }_{a p p}\right)$ correspond to electrolyses carried out at $E=1.2 \mathrm{~V}(\mathrm{vs} . \mathrm{Ag} / \mathrm{AgCl}, 3.0$ M).

When pseudo-first order kinetics is achieved (characterized by a logarithmic concentration decay along time) the slope of $k_{a p p}$ Vs. $j$ is given by $k_{R, H O} /\left(2 z \mathrm{~F} k_{O_{2}}\right)($ Eq. 
(13)). Values of $k_{R, H O} / k_{O_{2}}$ displayed in Table 3 were calculated from slopes of plots in Fig. 4.

Analysis of the intercept and slope contributions to $k_{a p p}$ can provide important insight on the reactivity of the species accordingly to the meaning of each parameters.

\subsection{Kinetic data analysis}

As discussed in sections 3.3 and 3.4 the rates of consumption of all species are much below the calculated values assuming mass transport control (Table 2), demonstrating that important kinetic hindrances are present.

Regarding the contribution of direct electron transfer reaction for the global oxidation, evaluated through the extrapolated $\left(k_{a p p}\right)_{j=0}$, the obtained values depended on the nature of species. Moreover, a correlation of 0.87 was observed between $\left(k_{a p p}\right)_{j=0}$ and the reciprocal of $E_{p}$. This serendipitous correlation can be explained considering that both variables are affected by a common parameter, i.e. the exchange current density $\left(j_{0}\right)$. Low $j_{0}$ values can be at the origin of high $E_{p}$ and simultaneously of low electron transfer rates. 4-HBA and 2,4-HBA display simultaneously the higher $E_{p}$ and the lower $\left(k_{a p p}\right)_{j=0}$, at the same time 2,3-HBA, 2,5-HBA, 3,4,5-HBA and HQ exhibit lower $E_{p}$ and have higher values of $\left(k_{a p p}\right)_{j=0}$. A better correlation between these two variables is difficult to attain since potential parameters reflect not only the kinetic but also the thermodynamic properties of a system [29], whereas $\left(k_{\text {app }}\right)_{j=0}$ reflects exclusively kinetic features.

The evaluated ratios $k_{R, H O} / k_{O_{2}}$ are listed in Table 3 along with structural representation of the HBA derivatives and with charge density values on the carbon atoms of the aromatic ring. The increase of $k_{R, H O} / k_{O_{2}}$ does not follow the number of $\mathrm{HO}$ groups of the molecule since the three most reactive species comprise a tri-, a mono- and a dihydroxybenzoic acid derivative. Alternatively, this kinetic parameter must be related to 
electronic properties of the species that can control reactions between $\mathrm{HO}$ radical and aromatic compounds. The presence of HO groups bonded to the aromatic ring induces negative charge densities on the carbon atoms of the ring. Considering the electrophilic nature of $\mathrm{HO}$ radical, the presence of negative charge densities on carbon atoms of the aromatic ring bonded to a hydrogen atom favours hydroxylation reaction resulting from HO radical electrophilic attack [30,31]. A relative reactivity order is proposed taking in consideration the number of negatively charged carbons as well as the magnitude of the charge density at positions where an $\mathrm{HO}$ group can be added (Table 3 ). The six compounds studied can be divided into three groups. The less reactive BA (with lower $k_{R, H O} / k_{O_{2}}$ ) exhibits positive charge densities at $\mathrm{C} 2$, C4 e $\mathrm{C} 6$ and slightly negative charge densities (almost null) at C3 and C5. The species 2,3-HBA and 2,5-HBA, more reactive than $\mathrm{BA}$ (higher $k_{R, H O} / k_{O_{2}}$ ), have one carbon with a significant negative charge density at C5 (-0.026) and C3 (-0.039), respectively. The most reactive species 3,4,5-HBA, 4-HBA and 2,4-HBA have two carbons with negative charge density. 3,4,5HBA, the less reactive of these three compounds, displays the lower charge densities ($0.010,-0.010$ ); 4-HBA (with higher $k_{R, H O} / k_{O_{2}}$ ) has intermediary charge densities ($0.039,-0.039)$ and $2,4-\mathrm{HBA}$ the most reactive species exhibits the highest charge densities $(-0.083,-0.063)$. 
Table 3: Values of $k_{R, H O} / k_{O_{2}}$ calculated for benzoic acid and for hydroxybenzoic acid derivatives from the slope of $k_{a p p}$ vs. $j$ using Eq. (13). Values of charge density are reported for carbon atoms from the aromatic ring that are bonded to a hydrogen atom. Negative values of charge density are in bold. $k_{R, H O} / k_{O_{2}}$

\begin{tabular}{|c|c|c|c|c|c|c|c|}
\hline & & $\mathrm{COOH}$ & $\mathrm{COOH}^{\mathrm{COH}}$ & COOH & $\mathrm{l}^{\mathrm{COOH}}$ & $\hat{l}^{\mathrm{COOH}}$ & $\left.\right|_{\mathrm{OH}} ^{\mathrm{COOH}}$ \\
\hline \multicolumn{8}{|c|}{$k_{R, H O} / k_{O_{2}}$} \\
\hline \multicolumn{8}{|c|}{$\left(10^{-3} \mathrm{~m}^{3} \mathrm{~mol}^{-1}\right)$} \\
\hline \multirow[t]{5}{*}{$\begin{array}{l}\text { Charge } \\
\text { density }\end{array}$} & $\mathrm{C} 2$ & 0.058 & & & -0.010 & 0.059 & \\
\hline & $\mathrm{C} 3$ & -0.002 & -0.039 & & & -0.039 & -0.083 \\
\hline & $\mathrm{C} 4$ & 0.049 & 0.014 & 0.008 & & & \\
\hline & $\mathrm{C} 5$ & -0.002 & & -0.026 & & -0.039 & -0.063 \\
\hline & C6 & 0.058 & 0.013 & 0.026 & -0.010 & 0.059 & 0.058 \\
\hline
\end{tabular}

\section{Conclusions}

Apparent rate constants were determined from the consumption of seven hydroxycontaining aromatic compounds. These values are much lower than the calculated considering mass transport control and depend on the nature of species, demonstrating that electrolyses rates are controlled by the kinetics of oxidation reactions. Values of $k_{a p p}$ were found to increase linearly with the current density of galvanostatic electrolysis with simultaneous $\mathrm{O}_{2}$ evolution. The intercept, $\left(k_{a p p}\right)_{j=0}$, of the linear dependence between $k_{a p p}$ and $j$ matches the apparent rate constant of potentiostatic electrolysis without $\mathrm{O}_{2}$ evolution. Thus, $\left(k_{a p p}\right)_{j=0}$ was deemed as a measure of the apparent rate constant of oxidation by direct electron transfer. From the slope of $k_{a p p}$ vs. $j$ values of $k_{R, H O} / k_{O_{2}}$ were determined for all species. These ratios of rate constants provide 
information concerning the reactivity of the species towards $\mathrm{OH}$ radicals. A good agreement between $k_{R, H O} / k_{O_{2}}$ values and the occurrence of negative charge densities on carbon atoms of the aromatic ring was observed. The consistency between the determined kinetic ratios and electronic properties of molecules substantiates the presented methodology for the kinetic study of oxidation by electrogenerated $\mathrm{HO}$ radical with simultaneous direct electron transfer.

\section{Acknowledgments}

Thanks are due to FCT (Fundação para a Ciência e Tecnologia) and FEDER (European Fund for Regional Development)-COMPETE-QREN-EU for financial support to the Research Centre, CQ/UM [PEst-C/QUI/UI0686/2011 (FCOMP-01-0124-FEDER022716)]. Raquel Oliveira thanks to FCT, POPH (Programa Operacional Potencial Humano) and FSE (Fundo Social Europeu) for the PhD Grant (SFRH/BD/64189/2009).

\section{References}

[1] M. Panizza, G. Cerisola, Application of diamond electrodes to electrochemical processes, Electrochimica Acta. 51 (2005) 191-199.

[2] M. Panizza, A. Kapalka, C. Comninellis, Oxidation of organic pollutants on BDD anodes using modulated current electrolysis, Electrochimica Acta. 53 (2008) 2289-2295.

[3] A. Kapalka, G. Fóti, C. Comninellis, Investigations of electrochemical oxygen transfer reaction on boron-doped diamond electrodes, Electrochimica Acta. 53 (2007) 1954-1961.

[4] F. Montilla, P.A. Michaud, E. Morallón, J.L. Vázquez, C. Comninellis, Electrochemical oxidation of benzoic acid at boron-doped diamond electrodes, Electrochimica Acta. 47 (2002) 3509-3513.

[5] M. Panizza, G. Cerisola, Direct And Mediated Anodic Oxidation of Organic Pollutants, Chemical Reviews. 109 (2009) 6541-6569.

[6] X. Zhu, M. Tong, S. Shi, H. Zhao, J. Ni, Essential Explanation of the Strong Mineralization Performance of Boron-Doped Diamond Electrodes, Environmental Science \& Technology. 42 (2008) 4914-4920. 
[7] A. Kapałka, G. Fóti, C. Comninellis, Kinetic modelling of the electrochemical mineralization of organic pollutants for wastewater treatment, Journal of Applied Electrochemistry. 38 (2008) 716.

[8] J. Iniesta, P.A. Michaud, M. Panizza, G. Cerisola, A. Aldaz, C. Comninellis, Electrochemical oxidation of phenol at boron-doped diamond electrode, Electrochimica Acta. 46 (2001) 35733578 .

[9] M. Mascia, A. Vacca, A.M. Polcaro, S. Palmas, J.R. Ruiz, A. Da Pozzo, Electrochemical treatment of phenolic waters in presence of chloride with boron-doped diamond (BDD) anodes: Experimental study and mathematical model, Journal of Hazardous Materials. 174 (2010) 314322.

[10] B. Louhichi, N. Bensalash, A. Gadri, Electrochemical Oxidation of Benzoic Acid Derivatives on Boron Doped Diamond: Voltammetric Study and Galvanostatic Electrolyses, Chemical Engineering \& Technology. 29 (2006) 944-950.

[11] T. Velegraki, G. Balayiannis, E. Diamadopoulos, A. Katsaounis, D. Mantzavinos, Electrochemical oxidation of benzoic acid in water over boron-doped diamond electrodes: Statistical analysis of key operating parameters, kinetic modeling, reaction by-products and ecotoxicity, Chemical Engineering Journal. 160 (2010) 538-548.

[12] Y. Cui, X. Li, G. Chen, Electrochemical degradation of bisphenol A on different anodes, Water Research. 43 (2009) 1968-1976.

[13] M. Panizza, G. Cerisola, Electrochemical degradation of gallic acid on a BDD anode, Chemosphere. 77 (2009) 1060-1064.

[14] C.A. Martinez-Huitle, F. Hernandez, S. Ferro, M.A.Q. Alfaro, A. de Battisti, Electrochemical oxidation: An alternative for the wastewater treatment with organic pollutants agents, Afinidad. 62 (2006) 26-34.

[15] C. Comninellis, Electrocatalysis in the electrochemical conversion/combustion of organic pollutants for waste water treatment, Electrochimica Acta. 39 (1994) 1857-1862.

[16] J.L. Wang, L.J. Xu, Advanced Oxidation Processes for Wastewater Treatment: Formation of Hydroxyl Radical and Application, Critical Reviews in Environmental Science and Technology. 42 (2011) 251-325.

[17] A. Kapalka, G. Fóti, C. Comninellis, G. Foti, The importance of electrode material in environmental electrochemistry: Formation and reactivity of free hydroxyl radicals on borondoped diamond electrodes, Electrochimica Acta. 54 (2009) 2018-2023.

[18] R. Oliveira, F. Bento, D. Geraldo, Aromatic hydroxylation reactions by electrogenerated HO radicals: A kinetic study, Journal of Electroanalytical Chemistry. 682 (2012) 7-13.

[19] R. Oliveira, J. Marques, F. Bento, D. Geraldo, P. Bettencourt, Reducing Antioxidant Capacity Evaluated by Means of Controlled Potential Electrolysis, Electroanalysis. 23 (2011) 692-700.

[20] K.E. Yakovleva, S.A. Kurzeev, E. V Stepanova, T. V Fedorova, B.A. Kuznetsov, O. V Koroleva, Characterization of plant phenolic compounds by cyclic voltammetry, Applied Biochemistry and Microbiology. 43 (2007) 661-668 LA - English.

[21] R. Liu, B. Goodell, J. Jellison, A. Amirbahman, Electrochemical Study of 2,3-Dihydroxybenzoic Acid and Its Interaction with $\mathrm{Cu}(\mathrm{II})$ and $\mathrm{H} 2 \mathrm{O} 2$ in Aqueous Solutions: Implications for Wood Decay, Environmental Science \& Technology. 39 (2004) 175-180. 
[22] S.M. Ghoreishi, M. Behpour, M. Khayatkashani, M.H. Motaghedifard, Simultaneous determination of ellagic and gallic acid in Punica granatum, Myrtus communis and Itriphal formulation by an electrochemical sensor based on a carbon paste electrode modified with multiwalled carbon nanotubes, Analytical Methods. 3 (2011) 636-645.

[23] M. Quan, D. Sanchez, M.F. Wasylkiw, D.K. Smith, Voltammetry of Quinones in Unbuffered Aqueous Solution: Reassessing the Roles of Proton Transfer and Hydrogen Bonding in the Aqueous Electrochemistry of Quinones, Journal of the American Chemical Society. 129 (2007) $12847-12856$.

[24] A.J. Bard, L.R. Faulkner, Electrochemical Methods: Fundamentals and Applications, 2nd ed., Wiley, New York, 2001.

[25] J.E. Baur, 19 - Diffusion Coefficients, in: C.G.Z.B.T.-H. of Electrochemistry (Ed.), Elsevier, Amsterdam, 2007: pp. 829-848.

[26] L.F. Ferreira, L.M. Souza, D.L. Franco, A.C.H. Castro, A.A. Oliveira, J.F.C. Boodts, et al., Formation of novel polymeric films derived from 4-hydroxybenzoic acid, Materials Chemistry and Physics. 129 (2011) 46-52.

[27] D. Nematollahi, H. Khoshsafar, Investigation of electrochemically induced Michael addition reactions. Oxidation of some dihydroxybenzene derivatives in the presence of azide ion, Tetrahedron. 65 (2009) 4742-4750.

[28] O. Simond, V. Schaller, C. Comninellis, Theoretical model for the anodic oxidation of organics on metal oxide electrodes, Electrochimica Acta. 42 (1997) 2009-2012.

[29] C. Amatore, Principles and Methods. Basic Concepts, in: M. Baizer, H. Lund (Eds.), Organic Electrochemistry, M. Dekker, New York, 1991: pp. 11-119.

[30] B. Lee, M. Lee, Prediction of Hydroxyl Substitution Site ( s ) of Phenol, Monochlorophenols and 4-Chloronitrobenzene by Atomic Charge Distribution Calculations, Bull. Korean Chem. Soc. 30 (2009) 4-7.

[31] S.M. Mukherji, S.P. Singh, R.P. Kapoor, R. Dass, Organic Chemistry, in: Organic Chemistry Vol. II, 2nd ed., New Age International, 2012: pp. 146-188. 